\title{
COMPRESSIVE SENSING FOR SENSOR CALIBRATION
}

\author{
Volkan Cevher and Richard Baraniuk \\ Electrical and Computer Engineering Department, \\ Rice University, Houston, TX 77005
}

\begin{abstract}
We consider a calibration problem, where we determine an unknown sensor location using the known track of a calibration target and a known reference sensor location. We cast the calibration problem as a sparse approximation problem where the unknown sensor location is determined over a discrete spatial grid with respect to the reference sensor. To achieve the calibration objective, low dimensional random projections of the sensor data are passed to the reference sensor, which significantly reduces the inter-sensor communication bandwidth. The unknown sensor location is then determined by solving an $\ell_{1}$-norm minimization problem (linear program). Field data results are provided to demonstrate the effectiveness of the approach.
\end{abstract}

Index Terms - Direction-of-arrival estimation; Microphone arrays; Sensor networks

\section{INTRODUCTION}

Acoustic arrays can estimate multiple source bearings by jointly processing the array microphone outputs along with knowledge of the microphone positions [1]. In turn, bearing estimates from multiple arrays (sensor nodes) with known locations and orientations can be used to triangulate the target positions on the ground plane. In this process, array calibration may imply the determination of the array signal manifold including the microphone locations, the unknown gain, phase, mutual-coupling coefficients $[2,3]$ or it may imply the determination of the sensor node location and orientation [4-6].

Conventionally, acoustic arrays are tethered with special deployment mechanisms for the individual microphones as unattended ground sensors (UGS). When they are deployed, the tethered mechanisms provide guidance for the array elements, making sure that the array elements are in their expected relative locations. Moreover, to realize the gains from the joint processing of array outputs, arrays are characteristically wired since the output data from each sensor in the array generally requires a high bandwidth for transmission. When this transmission is achieved in a wireless setting where sensors are battery powered, the sensor batteries can be quickly depleted and array elements may cause communication interference among themselves as they send relatively large data packets. Hence, because of their wired/tethered nature, arrays tend to have relatively small apertures and small number of elements, diminishing the main advantage derived from aperture gains and joint processing.

Recent advances in signal processing enable joint processing of microphone outputs over a wireless channel with minimal information exchange among the array elements $[7,8]$. These new methods exploit new compressive sensing (CS) ideas, which state that perfect recovery of

\footnotetext{
* Corresponding author (e-mail: volkan@ $@$ rice.edu). Prepared through collaborative participation in the Advanced Sensors Consortium sponsored by the U. S. Army Research Laboratory under the Collaborative Technology Alliance Program, Cooperative Agreement DAAD19-01-02-0008.
}

sparse sources may be obtained with high probability from highly undersampled data in the Nyquist-Shannon sense (see [9] and the references therein). A signal is called sparse if it contains only a small number of non-zero components in some transform domain, such as Fourier domain. In [7], a compressive wireless array (COWA) concept was introduced where quantized random projections of array element data are transmitted to obtain multiple target bearings. It is envisioned that COWAs can be randomly deployed over relatively larger apertures than conventional UGS. Hence, COWAs can obtain higher resolution bearing estimates as (i) their aperture size is not limited and (ii) they can seamlessly incorporate more array elements for joint processing.

New calibration algorithms are needed for COWAs to determine the sensor locations after the deployment. In this paper, we consider the localization of individual microphone positions of a COWA using a known calibration source. We assume that the sensors already have their gain and phase information calibrated. Moreover, we assume that their mutual coupling affects are small. We provide a sparse approximation for sensor location estimation where we assume that the unknown sensor locations can only occur over a discrete grid. In spirit, our approach is similar to the discrete approximations of the state posterior density in particle filters [10]. We propose a discrete set of locations for the unknown sensor location and calculate their weights by an $\ell_{1}$-minimization problem, which can be efficiently solved using a linear program. The key idea is that the target location is sparse over the proposed discrete locations. We show that by using the knowledge of a target track, low dimensional random projections (or random samples) of the target data vectors at the array elements can be used to calibrate the array element locations with respect to a reference sensor.

Compared to the relevant UGS calibration work in the literature $[2,3]$, our approach is fundamentally different as we provide a sparse approximation for the sensor locations as opposed to continuous solutions. Due to sparsity in the problem, we do not need to transmit the full array data to achieve calibration. Hence, our approach is more suitable for communication constrained settings, such as wireless operations for simultaneous tracking and calibration [11]. Moreover, the calibration of each sensor location can be done in parallel as their computations are independent of each other. By the mathematical formulation, our calibration approach is the dual solution of our localization via spatial sparsity approach, which determines multiple target locations using the known sensor positions [12].

The organization of the paper is as follows. Section 2 reviews the basic CS concepts that are relevant to our problem. Section 3 explains our sparse approximation for the calibration problem and its solution. Field experiments are given in Sect. 4 to demonstrate the effectiveness of the proposed approach. 


\section{COMPRESSIVE SENSING BACKGROUND}

\subsection{Sparse Representations}

Suppose that we have a vector $z$ of size $N \times 1$. The $n$th element of the vector $z$ is referred to as $z[n]$, where $n=1, \ldots, N$. Let us assume that the basis $\boldsymbol{\Psi}=\left[\boldsymbol{\psi}_{1}, \ldots, \boldsymbol{\psi}_{N}\right]$ provides a $K$-sparse representation of $\boldsymbol{z}$ :

$$
\boldsymbol{z}=\sum_{n=1}^{N} \theta[n] \boldsymbol{\psi}_{n}=\sum_{l=1}^{K} \theta\left[n_{l}\right] \boldsymbol{\psi}_{n_{l}},
$$

where $\theta[n]$ is the coefficient of the $n$th basis vector $\psi_{n}\left(\psi_{n}: N \times 1\right)$ and the coefficients indexed by $n_{l}$ are the $K$ nonzero entries of the basis decomposition. Equation (1) can be more compactly expressed as follows

$$
z=\Psi \theta
$$

where $\boldsymbol{\theta}$ is an $N \times 1$ column vector with $K$-nonzero elements. Using $\|\cdot\|_{p}$ to denote the $\ell_{p}$ norm where the $\ell_{0}$ norm simply counts the nonzero elements of $\boldsymbol{\theta}$, we call a vector $\boldsymbol{z}$ as $K$-sparse if $\|\boldsymbol{\theta}\|_{0} \leq K$. The matrix $\Psi$ is called the sparsity basis.

In the sensor calibration problem, the sensor location can be ideally modelled as 1-sparse vector on a discrete grid that includes the sensor location. However, in most cases, the approximation grid does not include the sensor location and the sensors take noisy measurements. Hence, a sparse approximation for the sensor location does not result in an exactly 1-sparse representation; instead, it has a few non zero coefficients that decay to zero. The discussion below also applies to such cases. We further discuss the sparse approximation of the sensor locations in Sect. 3.

\subsection{Random/Incoherent Projections}

In the CS framework, we assume that the $K$-largest $\theta[n]$ are not measured directly. Rather, $M<N$ linear projections of the vector $\boldsymbol{z}$ onto another set of vectors $\boldsymbol{\Phi}=\left[\phi_{1}^{\prime}, \ldots, \phi_{M}^{\prime}\right]^{\prime}$ are measured or communicated:

$$
\boldsymbol{y}=\boldsymbol{\Phi} z=\Phi \Psi \theta,
$$

where the vector $y(M \times 1)$ constitutes the compressive samples and the matrix $\boldsymbol{\Phi}(M \times N)$ is called the measurement matrix. Since $M<N$, recovery of the vector $\boldsymbol{z}$ from the compressive samples $\boldsymbol{y}$ is underdetermined; however, as we discuss below, the additional $K$-sparsity assumption makes recovery possible.

The CS theory states that when (i) the columns of the sparsity basis $\Psi$ cannot sparsely represent the rows of the measurement matrix $\boldsymbol{\Phi}$ and (ii) the number of measurements $M$ is greater than $\mathcal{O}\left(K \log \left(\frac{N}{K}\right)\right)$, then it is possible to recover the set of nonzero entries of $\boldsymbol{\theta}$ from $\boldsymbol{y}[13,14]$. Then, the vector $\boldsymbol{z}$ can be obtained by the linear transformation of $\boldsymbol{\theta}$ in (1). The first condition is called the incoherence of the two bases and it holds for many pairs of bases, e.g., delta spikes and the sine waves of the Fourier basis. Surprisingly, the incoherence also holds with high probability between an arbitrary basis and a randomly generated one, e.g., i.i.d. Gaussian or Bernoulli/Rademacher \pm 1 vectors.

\subsection{Signal Recovery via $\ell_{1}$ Optimization}

There exists a computationally efficient recovery method for $\boldsymbol{\theta}$ given $\boldsymbol{y}$ based on the following $\ell_{1}$-optimization problem $[13,14]$ :

$$
\widehat{\boldsymbol{\theta}}=\arg \min \|\boldsymbol{\theta}\|_{1} \text { s. t. } \boldsymbol{y}=\boldsymbol{\Phi} \boldsymbol{\Psi} \boldsymbol{\theta} .
$$

This optimization problem, also known as Basis Pursuit [14], can be efficiently solved using polynomial time algorithms.
Other formulations are used for recovery from noisy measurements such as the Lasso and basis pursuit with quadratic constraint [13]. In this paper, we use Basis Pursuit Denoising (BPDN) for recovery:

$$
\widehat{\boldsymbol{\theta}}=\arg \min \|\boldsymbol{\theta}\|_{1}+\frac{1}{2} \beta\|\boldsymbol{y}-\boldsymbol{\Phi} \boldsymbol{\Psi} \boldsymbol{\theta}\|_{2}^{2},
$$

where $0<\beta<\infty[15]$.

\section{SPARSE APPROXIMATION FOR THE CALIBRATION PROBLEM}

In the calibration problem, our objective is the determine the unknown sensor location with respect to a known sensor location, denoted as the reference sensor (RS). Suppose that we restrict the unknown sensor locations to lie on a discrete grid of points $\zeta=\left\{\zeta_{i} \mid i=0, \ldots, N ; \zeta_{i}=\right.$ $\left.\left[\zeta_{x i}, \zeta_{y i}\right]^{\prime}\right\}$, where $\zeta_{0}$ is the 2D location of the RS. Define a grid selector vector $\boldsymbol{\theta}$, which is $K$ sparse in the $N$ dimensional location space where $K \ll N$. The vector $\boldsymbol{\theta}$ over the grid $\boldsymbol{\zeta}$ is the focus of the paper.

We assume that we have a calibration target with known position track $\chi_{t}=\left[\chi_{x}(t), \chi_{y}(t)\right]$ at times $t=t_{1}, \ldots, t_{L}$. The RS receives noisy acoustic measurements of the delayed and attenuated target signal as follows

$$
z_{0}(t)=\frac{1}{R_{0}(t)} s\left(t-\frac{R_{0}(t)}{c}\right)+n_{0}(t),
$$

where $z_{0}(t)$ is the RS signal, $s(t)$ is the unknown calibration target signal, $c$ is the acoustic propagation speed, $n_{0}(t)$ is an additive noise, and

$$
R_{0}(t)=\sqrt{\left(\chi_{x}(t)-\zeta_{x 0}\right)^{2}+\left(\chi_{y}(t)-\zeta_{y 0}\right)^{2}} .
$$

In (6), we assume an isotropic medium with spherical propagation and ignore the Doppler effects due to the time retardation effects.

Similarly, the sensor, whose position is unknown (denoted as unknown sensor- US), receives noisy measurements $z(t)$ of the target signal at different delay and attenuation, which depends on its position $\zeta$. An estimate of this sensor signal can be made over the grid locations $\zeta$ using (6):

$$
z_{i}(t)=\frac{R_{0}(t)}{R_{i}(t)} z_{0}\left(t+\frac{R_{0}(t)}{c}-\frac{R_{i}(t)}{c}\right),
$$

where $R_{i}(t)$ inherits its definition from (7). Now, the US signal can be represented as a sparse combination of $z_{i}(t)$ 's. In fact, if a grid point $j$ precisely coincides with the US location, then we have $z(t)=z_{j}(t)$. This assumes that $z(t)$ cannot be represented as a linear combinations of other grid points. This assumption is satisfied for signals that decorrelate at small lags, such as vehicle signals and human speech.

Define a vector $\boldsymbol{z}_{i}=\left[z_{i}\left(t_{1}\right), \ldots, z_{i}\left(t_{L}\right)\right]^{\prime}$, consisting of the concatenation of the estimated signal samples via (8) over the grid locations, and form a localization matrix $\boldsymbol{\Psi}$, whose $j$ column is $\boldsymbol{z}_{j}$. Then, we have the following relation for the US signal:

$$
z=\Psi \theta \text {. }
$$

Instead of sending the full received measurements, the US sends the compressive measurements $\boldsymbol{y}$ to the RS using a random measurement matrix $\boldsymbol{\Phi}$ that is incoherent with $\Psi$ :

$$
y=\Phi z=\Phi \Psi \theta
$$

In our problem, we use vehicles as calibration targets. Hence, the columns of $\Psi$ consist of frequency sparse signals. Then, for $\boldsymbol{\Phi}$, we can either use a random matrix or just a subset of simple permuted delta functions, corresponding to sending randomly chosen samples of the received signal. Our approach is summarized in Table 1. 


\section{Table 1. Sensor Location Calibration}

Given the observed data $z_{0}$ at the RS and its location $\zeta_{0}$, the compressive measurements $y$ from the US, and a grid $\zeta$, do

1. Create the localization matrix $\Psi$ using the grid locations, the RS location, and (8).

2. Solve the BPDN problem (5).

3. Repeat this process for other unknown sensor locations.

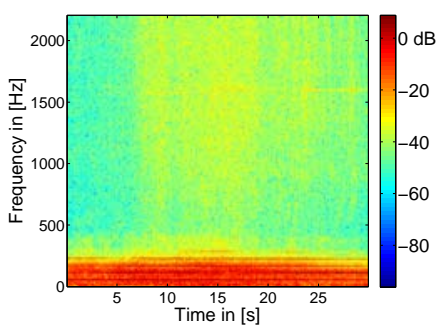

(a)

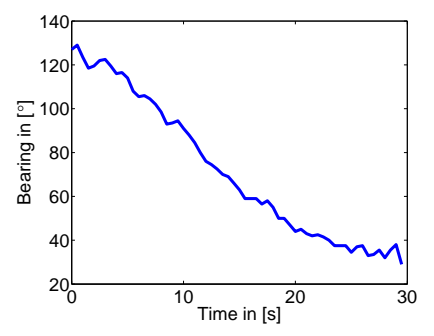

(b)
Fig. 1. (a) Time-frequency plot of the received acoustic data at RS is shown. The spectral sparsity of the source is not exploited in our calibration solution. (b) Compressive wireless array processing results with 10 bit communication message size per bearing from each 9 microphones.

\section{EXPERIMENTS}

A uniform circular acoustic array with 10 microphones (9 microphones on the perimeter with 1.44 meter radius and one at the center) is used to collect the acoustic data. The center microphone is chosen as the RS. The acoustic data sampling rate at RS is $F_{s}=4410 \mathrm{~Hz}$. A single target recording is obtained for $30 \mathrm{~s}$. In the test, the target is moving with a constant speed of $20 \mathrm{mph}$.

We used the COWA formulation in [7] to estimate the bearing track of the target. To estimate bearings, the microphone array elements send the sign of the compressive measurements (size $10 \times 1$ ) of their received data (size $2205 \times 1$ ) to the RS twice per second. A total of $2 \times 9 \times$ $10=180$ bits from all US are transmitted to the RS sensor per second $(0.176 \mathrm{~KB} / \mathrm{s}$ bandwidth at the RS). The RS then calculates two bearings of the target per second using the signal recorded at $F_{s}$ and the compressive measurements from the other array elements. The spectral lines of the target calculated at the RS and the estimated bearing track are shown in Fig. 1.

To demonstrate the calibration idea, we used the target bearing track (denoted as $\phi(t)$ measured counterclockwise with respect to the $x$-axis) in Fig. 1(b) as the ground truth track for the target location. Since the target is in the far field of the array, sensor signal estimates over the grid points in (8) are approximated as follows:

$$
\begin{aligned}
z_{i}(t) & =\frac{R_{0}(t)}{R_{i}(t)} z_{0}\left(t+\frac{R_{0}(t)}{c}-\frac{R_{i}(t)}{c}\right) \\
& \approx z_{0}\left(t+\tau_{0 i}(t)\right)
\end{aligned}
$$

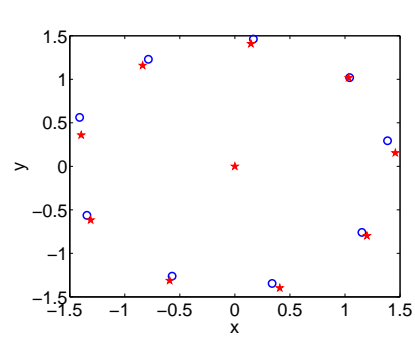

(a)

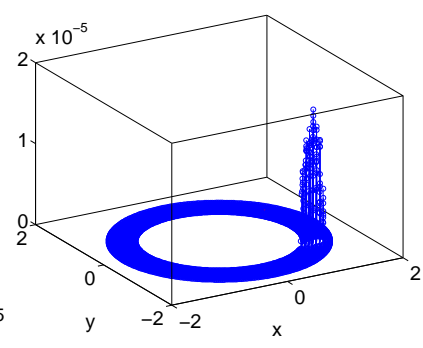

(b)
Fig. 2. (a) Field calibration results on a regular polar grid. True locations are shown with stars whereas the estimated locations are marked with circles. (b) Resulting sparse approximation for a sensor location.

where the relative time delay is expressed as

$$
\tau_{0 i}(t)=\frac{r_{0 i}}{c} \cos (\varphi-\phi(t))
$$

using the polar coordinates of the $i$ th sensor with respect to the RS

$$
\begin{array}{r}
r_{0 i}=\sqrt{\left(\zeta_{x i}-\zeta_{x 0}\right)^{2}+\left(\zeta_{y i}-\zeta_{y 0}\right)^{2}}, \\
\varphi_{0 i}=\tan ^{-1}\left(\frac{\zeta_{y i}-\zeta_{y 0}}{\zeta_{x i}-\zeta_{x 0}}\right) .
\end{array}
$$

Figure 2(a) illustrates the calibration results from the 30s target data. During the calibration time, the each of the 9 US sensors send a total of $M=$ 132 random data points (1000: 1 data compression) each at 64 bit quantization, totalling $9 \times 132 \times 64=76032$ bits data communication over 30 seconds $(2.475 \mathrm{~KB} / \mathrm{s}$ rate at the RS). The error in localization for each microphone is $[15.85,6.02,8.60,5.53,6.65,20.25,8.78,5.92,0.89] \mathrm{cm}$ with an average of $8.72 \mathrm{~cm}$ (approximately $3 \%$ of the array aperture).

To obtain the results above, a regular polar grid between the ranges $1.25 \mathrm{~m}$ and $1.65 \mathrm{~m}$ with $50 \mathrm{~cm}$ resolution is used along with an angle resolution of 1 degree, totaling $N=3240$. In general, we can decrease the grid size and increase the number of compressive measurements to obtain the same level of estimation accuracy. To illustrate this idea, we created a uniformly random grid on a ring with $0.5 \mathrm{~m}$ inner and $2 \mathrm{~m}$ outer radii with $N=500$ grid points. In this case, we increased the number of total compressive samples 10 fold to $M=1320$. The localization error for each microphone becomes $[17.30,5.76,4.43,3.75,6.26,25.83,7.02,3.96,3.78] \mathrm{cm}$ with an average of $8.67 \mathrm{~cm}$. Hence, the accuracy of the localization not only depends on the number of communicated data samples, but also the resolution of the grid points. For the interested reader, an analysis of the minimum possible grid spacing for localization estimates is given in [12].

To test whether the ground truth errors make a difference, we obtained frequency adaptive beamforming estimates of the target track using the full data from all the sensors, requiring a bandwidth of $2.4225 \mathrm{MB} / \mathrm{s}$ compared to the COWA approach, which uses $0.176 \mathrm{~KB} / \mathrm{s}$ bandwidth. The bearing estimation results for the ground truth are shown in Fig. 4 along with the compressive wireless array results (COWA) from Fig. 1(b). In this case, the localization error for each microphone becomes $[13.35,10.35,3.54,2.52,5.05,20.70,4.09,7.46,0.44] \mathrm{cm}$ with an average of $7.50 \mathrm{~cm}$. In the estimation, $M=132$ samples are used. Compared to the estimation results in Fig. 2(a), there is a $1.22 \mathrm{~cm}$ improvement in the average localization error due to the improved ground truth estimate.

Our experimental results are summarized in Table 2. 
Table 2. Summary of results

\begin{tabular}{||c|c|c|c|c|c|}
\hline \hline Ground Truth & Grid Type & Number of Grid Points $N$ & $M$ & Bandwidth @ RS & Average Localization Error \\
\hline \hline COWA & Uniform & 3240 & 132 & $\approx 2.5 \mathrm{~KB} / \mathrm{s}$ & $8.72 \mathrm{~cm}$ \\
COWA & Random & 500 & 1320 & $\approx 25 \mathrm{~KB} / \mathrm{s}$ & $8.67 \mathrm{~cm}$ \\
MVDR & Uniform & 3240 & 132 & $\approx 2.5 \mathrm{~KB} / \mathrm{s}$ & $7.50 \mathrm{~cm}$ \\
\hline \hline
\end{tabular}

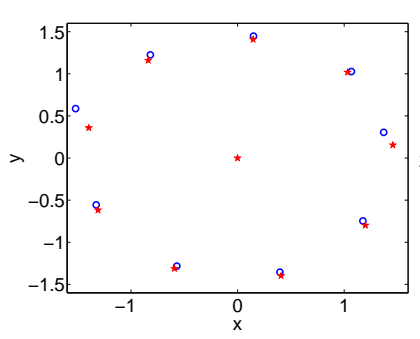

(a)

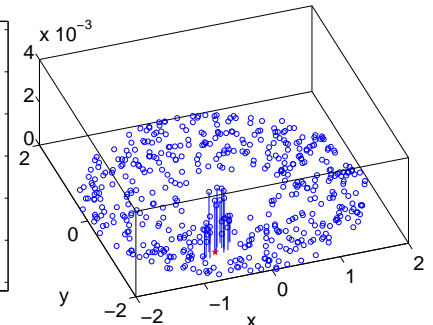

(b)
Fig. 3. (a) Field calibration results with a random grid. True locations are shown with stars whereas the estimated locations are marked with circles. (b) Resulting sparse approximation for a sensor location.

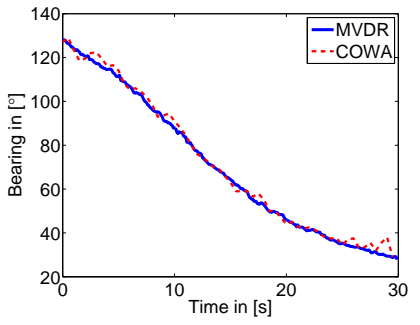

(a)

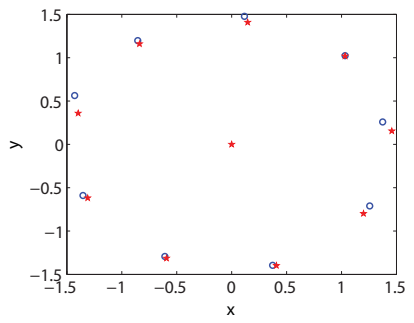

(b)
Fig. 4. (a) Bearing estimation results with the full acoustic data from all the sensors using a frequency adaptive MVDR algorithm. For comparison, the compressive wireless array bearing estimation result is superposed with the dashed lines. (b) Field calibration result with the MVDR ground truth estimates on the same grid used in Fig. 2.

\section{CONCLUSIONS}

Recent advances in bearing estimation methods allows for randomly deployed sensors to estimate multiple target bearings wirelessly $[7,8]$. The thrust in these new methods are achieved through a new signal processing concept called the compressive sensing. Because the wireless operations are constrained by sensor batteries, there is now an increasing need for calibration algorithms that require low communication bandwidth. In this paper, we have demonstrated that the CS ideas can be used for the array location calibration with minimal intersensor information exchange, which makes the presented calibration algorithm suitable for battery operated wireless sensor networks. For our method, we demonstrated through experiments that the estimation accuracy depends on the sparse approximation grid resolution, the veracity of the ground truth estimates, as well as the number of compressive measurements. Although our approach used a sparse approximation for the sensor locations, the resulting estimate is a continuous estimate since it is a weighted average of the sparse grid locations. Future work will focus more on the theoretical aspects of the calibration problem using CS.

\section{REFERENCES}

[1] D. H. Johnson and D. E. Dudgeon, Array Signal Processing: Concepts and Techniques, Prentice Hall, 1993.

[2] B. C. Ng and C. M. S. See, "Sensor array calibration using a maximum-likelihood approach," IEEE Trans. on Antennas and Propagation, vol. 44, pp. 827-835, June 1996.

[3] B. Friedlander and A. J. Weiss, "Direction finding in the presence of mutual coupling," IEEE Transactions on Antennas and Propagation, vol. 39, no. 3, pp. 273-284, 1991.

[4] V. Cevher and J. H. McClellan, "Sensor array calibration via tracking with the extended Kalman filter," in Proc. of the the Fifth Ann. Fed. Lab. Symp. on Adv. Sensors, College Park, MD, 20-22 March 2001, pp. 51-56.

[5] R. L. Moses, D. Krishnamurthy, and R. Patterson, "An autocalibration method for unattended ground sensors," EURASIP Journal on Applied Signal Processing, vol. 4, pp. 348-358, 2003.

[6] A. T. Ihler, J. W. Fisher III, R. L. Moses, and A. S. Willsky, "Nonparametric belief propagation for self-localization of sensor networks," IEEE Journal on Selected Areas in Communications, vol. 23, no. 4, pp. 809-819, 2005.

[7] V. Cevher, A. C. Gurbuz, J. H. McClellan, and R. Chellappa, "Compressive wireless arrays for bearing estimation," in ICASSP 2008, Las Vegas, Nevada, March 30 -April 42008.

[8] A. C. Gurbuz, V. Cevher, and J. H. McClellan, "A compressive beamformer," in ICASSP 2008, Las Vegas, Nevada, March 30 April 42008.

[9] R. G. Baraniuk, "Compressive Sensing," IEEE Signal Processing Magazine, vol. 24, no. 4, pp. 118-121, 2007.

[10] M. S. Arulampalam, S. Maskell, N. Gordon, and T. Clapp, "A tutorial on particle filters for online nonlinear/non-Gaussian Bayesian tracking," IEEE Trans. on Signal Processing, vol. 50, no. 2, pp. 174-188, 2002.

[11] C. Taylor, A. Rahimi, J. Bachrach, H. Shrobe, and A. Grue, "Simultaneous localization, calibration, and tracking in an ad hoc sensor network," in IPSN. 2006, pp. 27-33, ACM Press New York, NY, USA.

[12] V. Cevher, M. Duarte, and R. G. Baraniuk, "Distributed Target Localization via Spatial Sparsity," in European Signal Processing Conference (EUSIPCO), Lausanne, Switzerland, Aug 2008.

[13] E. Candes, "Compressive sampling," in Proceedings of the International Congress of Mathematicians, 2006.

[14] D. L. Donoho, “Compressed Sensing," IEEE Transactions on Information Theory, vol. 52, no. 4, pp. 1289-1306, 2006.

[15] S. S. Chen, D. L. Donoho, and M. A. Saunders, "Atomic Decomposition by Basis Pursuit," SIAM Journal on Scientific Computing, vol. 20, pp. 33, 1998. 